\title{
Verbal Instruction Reduces Patellofemoral Joint Loading During Bodyweight Squatting
}

\author{
Thomas G. Almonroeder, Emily Watkins, and Tricia Widenhoefer
}

\begin{abstract}
Context: The bodyweight squat exercise is a common component for treatment and prevention of patellofemoral pain; however, it can also place a high load on the patellofemoral joint. Restricting anterior motion of the knees relative to the toes during squatting appears to reduce patellofemoral loading. However, exercise professionals typically rely on verbal instructions to alter squat technique. Objective: To evaluate the influence of verbal instructions regarding squat technique on patellofemoral joint loading. Design: Cross-sectional study. Setting: Motion analysis laboratory. Participants: Eleven uninjured females. Intervention: Participants performed bodyweight squats before (baseline) and after receiving verbal instructions to limit anterior knee motion. Two different types of verbal instruction were used, one intended to promote an internal focus of attention and the other intended to promote an external focus of attention. Three-dimensional kinematics and kinetics were recorded using a multicamera system and force plate. Main Outcome Measures: Sagittal plane patellofemoral joint forces and stress were estimated using a musculoskeletal model. Results: Participants demonstrated a reduction in patellofemoral joint forces ( $35.4 \mathrm{vs} 31.3 \mathrm{~N} / \mathrm{kg} ; P=.01$ ) and stress (10.7 vs $9.2 \mathrm{mPa} ; P=.002)$ after receiving instructions promoting an internal focus of attention, compared with their baseline trials. Participants also demonstrated a reduction in patellofemoral joint forces ( $35.4 \mathrm{vs} 32.3 \mathrm{~N} / \mathrm{kg} ; P=.03$ ) and stress $(10.7$ vs $9.6 \mathrm{mPa} ; P=.04)$ after receiving instructions promoting an external focus of attention (vs baseline). However, there were no significant differences in patellofemoral forces $(P=.84)$ or stress $(P=.41)$ for trials performed with an internal versus external attentional focus. Conclusion: It appears that verbal instruction regarding knee position influences patellofemoral joint loading during squatting.
\end{abstract}

Keywords: biomechanics, attention, sports medicine

Patellofemoral pain is relatively common in young, active females. ${ }^{1,2}$ Unfortunately, the presence of patellofemoral pain during adolescence and/or early adulthood can limit participation in physical activity ${ }^{3}$ and appears to be associated with the development of future patellofemoral joint osteoarthritis. ${ }^{4,5}$ As a result, improving our ability to prevent and treat the disorder is imperative to promoting long-term health.

The pathogenesis of patellofemoral pain is complex; however, elevated patellofemoral joint forces (PFJFs) and patellofemoral joint stress (PFJS), that is, the forces relative to the joint contact area, appear to be contributing factors. ${ }^{6-9}$ Weight-bearing lowerextremity strengthening exercises, such as the bodyweight squat, are recommended for managing patellofemoral pain. ${ }^{10}$ A potential benefit of weight-bearing exercises is that they may be more "functional" than nonweight-bearing exercises, as they more adequately reflect the demands imposed by activities such as running, landing, and so on. ${ }^{11}$ However, weight-bearing exercises, such as the squat, can also place a substantial load on the patellofemoral joint. ${ }^{12}$ As a result, there is a need to consider how manipulating squat technique may influence PFJFs and PFJS to help ensure that individuals experience the potential benefits of the exercise without predisposing themselves to patellofemoral pain or exacerbating their symptoms.

The position of the knees relative to the toes is often a key point of interest when analyzing squat technique. ${ }^{13-15} \mathrm{~A}$ common belief is that allowing the knees to translate forward in excess

The authors are with Physical Therapy Program, Rinker-Ross School of Health Sciences, Trine University, Fort Wayne, IN, USA. Almonroeder (almonroedert@ trine.edu) is corresponding author. during squat performance increases the forces and stress on the knee. Results reported by Kernozek et $\mathrm{al}^{16}$ appear to support this common belief, as they found that PFJFs and PFJS were greater when young females performed bodyweight squats where their knees were required to move past their toes, compared with squats where their knees needed to remain behind their toes. Guidewires were used to direct/restrict knee motion during the squat trials for both conditions (ie, knees past toes and knees behind toes). These results appear to support the recommendation that individuals should limit anterior knee motion when squatting. However, exercise professionals often rely on verbal instruction to alter squat technique (vs physical restrictions). As a result, it is important to explore how verbal instructions, such as those utilized by exercise professionals, influence patellofemoral joint loading during bodyweight squatting.

Verbal instructions regarding exercise technique can be slightly modified to promote either an internal focus of attention (FoAin) or an external focus of attention (FoAex). ${ }^{17,18}$ Instructions intended to promote an FoAin direct individuals to monitor their own movement (eg, "while squatting, try to prevent your knees from going past your toes"), whereas instructions intended to promote an FoAex guide individuals to focus on the effects of their movement (eg, "squat like you are sitting back into a chair"). Exercise professionals appear to typically adopt instructions that promote an FoAin, ${ }^{19,20}$ despite findings indicating that an FoAex may promote superior performance for a wide range of movement tasks. ${ }^{21-25}$ However, to our knowledge, there has not been an attempt to compare the influence of squat instructions intended to promote an FoAin versus FoAex. An analysis of this nature may help to determine if different types of verbal instruction elicit more/ less optimal mechanical responses. 
The purpose of this study was to investigate how verbal instruction influences patellofemoral joint loading during bodyweight squatting. We hypothesized that participants would demonstrate a reduction in PFJFs and PFJS when they were given instructions aimed at limiting anterior knee motion during squatting. In addition, we also wanted to compare the influence of different types of verbal instruction (ie, those intended to promote an FoAin vs FoAex). We hypothesized that we would observe greater reductions in PFJFs and PFJS when participants were given instructions intended to promote an FoAex, compared with trials where they had been instructed to adopt an FoAin. The results of our study may provide valuable insight to professionals involved in exercise prescription for the treatment and prevention of patellofemoral pain.

\section{Methods}

\section{Participants}

This cross-sectional study included 11 females aged between 18 and 35 years. At minimum, participants were required to engage in weight-bearing aerobic exercise at least 3 times per week for a total of 60 minutes. Individuals were excluded from this study if they had a history of significant injury (eg, ligament tear, fracture) or surgery involving their back or lower-extremities, or any injury in the previous 6 months that limited their activity. We performed an a priori sample size estimate using an alpha of .05, a beta of 0.20 , and a within-factor correlation of .7. Anticipating a medium to large effect size $\left(\eta_{\mathrm{p}}^{2}=.10\right),{ }^{26}$ this estimate indicated that 11 participants would ensure adequate power for our omnibus test (repeatedmeasures analysis of variance [ANOVA]). We used G*Power software (version 3.1; University of Düsseldorf, Düsseldorf, Germany) for sample size estimation. ${ }^{27}$ The study was approved by the Institutional Review Board at Trine University, and all participants provided informed consent.

\section{Testing Protocol}

In general, the testing protocol involved participants performing bodyweight squats before and after receiving instructions that promoted an FoAin or FoAex. Three-dimensional kinematics and forces were recorded using a multicamera motion capture system and force plate to estimate patellofemoral loading during the squat trials.

Participants were provided with cross-trainer shoes to wear during the session (Avi-Rival, AVIA; Sequential Brands Group Inc, New York, NY). Retroreflective markers were placed bilaterally on the iliac crests, anterior superior iliac spines, posterior superior iliac spines, and greater trochanters, as well as on the medial/lateral epicondyles, medial/lateral malleoli, and the first and fifth metatarsal heads of the dominant limb (ie, the leg used to kick a ball). Additional marker clusters were adhered to the thigh, shank, and heel counter of the shoe. These marker clusters were used to track the thigh, shank, and foot segments during the movement trials. The pelvis was tracked by the markers placed on the posterior superior iliac spines and on the iliac crests. With the markers in place, a standing calibration trial was captured. The 3-dimensional positions of the retroreflective markers were recorded at $200 \mathrm{~Hz}$ using an 8-camera motion capture system (Vicon Motion Systems, Inc, Oxford, United Kingdom).

Following the calibration trial, participants completed 10 bodyweight squats as a warm-up. Then, they performed 5 additional bodyweight squats (baseline trials) with the foot of their dominant limb positioned on a force plate that recorded 3-dimensional ground reaction forces at $1000 \mathrm{~Hz}$ (OR6-7-2000; Advanced Mechanical Technology, Inc, Watertown, MA). Participants were instructed to squat to a depth where their thighs were approximately parallel to the ground. Squat depth was monitored visually throughout testing to ensure consistency among trials and conditions. Participants were allowed to choose their own foot position (ie, stance width, angle of inward/outward rotation); however, they were required to maintain this foot position throughout testing. We outlined the position of the participant's foot using tape and asked them to limit foot movement between trials/conditions to ensure that their foot position was maintained. We cued participants to keep their arms parallel to the ground with their elbows extended during performance of the squat trials. Participants were also required to pause briefly between trials so that the squats were performed as distinct movements.

Once the baseline trials were complete, participants performed 5 additional squat trials after receiving verbal instructions intended to promote either an FoAin or FoAex. Prior to the FoAin trials, participants were given the following instructions: "while squatting, try to prevent your knees from going past your toes." Prior to the FoAex trials, they were instructed as follows: "squat like you are sitting back into a chair." These instructions were slightly modified versions of examples provided by Benjaminse et al. ${ }^{28}$ Instructions were provided once prior to initiation of the squat trials for each condition. Participants performed 5 trials for both the FoAin and the FoAex conditions. This resulted in 15 total trials ( 5 baseline, 5 FoAin, and 5 FoAex). The trials for each condition were performed consecutively (ie, participants did not alternate between FoAin and FoAex trials). The order of the FoAin and FoAex conditions was counterbalanced by alternating the testing order (FoAin, FoAex vs FoAex, FoAin) as participants were enrolled in the study. Six participants performed the FoAin condition first, and 5 participants performed the FoAex condition first.

\section{Preliminary Data Processing}

The marker and ground reaction force data were filtered using a fourth-order, zero-lag, recursive Butterworth filter with a cutoff frequency of $20 \mathrm{~Hz}$. Right-handed Cartesian local coordinate systems were defined to describe the position and orientation of the pelvis, thigh, leg, and foot. The midpoint between the medial/ lateral epicondyle markers and the medial/lateral malleoli markers were used to define the knee and ankle joint centers, respectively. The markers on the anterior superior iliac spines were used to define the hip joint center. ${ }^{29,30} \mathrm{~A}$ joint coordinate system approach was used to calculate the 3 -dimensional joint angles. ${ }^{31}$ Net joint moments were calculated using a Newton-Euler approach and resolved into the local coordinate system of the distal segment. Body segment parameters were based on cadaver data. ${ }^{32}$ Preliminary data processing was performed using Visual3D software (C-Motion Inc, Rockville, MD).

\section{Patellofemoral Joint Model}

The knee-flexion angles and the net internal knee extension moments were input into a model to estimate the PFJFs and the PFJS. The quadriceps effective moment arm $(r)$ was estimated as a function of the knee-flexion angle $(x)$ using a third-order polynomial equation developed from data reported by van Eijden et $\mathrm{al}^{33}$ (Equation 1). The quadriceps force (QF) was estimated by 
dividing the knee extension moment $\left(M_{\text {ext }}\right)$ by this moment arm (Equation 2).

$$
\begin{gathered}
r=8.0 \mathrm{e}^{-5} x^{3}-0.013 x^{2}+0.28 x+0.046, \\
\mathrm{QF}=M_{\text {ext }} / r .
\end{gathered}
$$

A constant $(k)$ was calculated as a function of the knee-flexion angle $(x)$ using an equation reported by Brechter and Powers ${ }^{6}$ (Equation 3). This constant reflects the proportion of the QFs acting at the patellofemoral joint. The PFJFs was estimated by multiplying this constant by the QF (Equation 4).

$$
\begin{gathered}
k=\left(-3.84 \mathrm{e}^{-5} x^{2}+1.47 \mathrm{e}^{-3} x+0.462\right) /\left(-6.98 \mathrm{e}^{-7} x^{3}\right. \\
\left.+1.55 \mathrm{e}^{-4} x^{2}-0.0162 x+1\right), \\
\text { PFJFs }=\mathrm{QF} \times k .
\end{gathered}
$$

The patellofemoral joint contact area (PFJCA) was estimated as a function of the knee-flexion angle $(x)$ using a second-order polynomial equation developed from data reported by Powers et $\mathrm{al}^{34}$ (Equation 5), and the PFJS was calculated by dividing the PFJFs by the PFCA (Equation 6). The PFJS is reported in units of millipascals $(\mathrm{mPa})$.

$$
\begin{gathered}
\text { PFJCA }=-0.028 x^{2}+4.17 x+70.81, \\
\text { PFJS }=\text { PFJFs } / \text { PFJCA. }
\end{gathered}
$$

All calculations involved in the patellofemoral joint model were performed using a custom MATLAB script (MathWorks, Inc, Natick, MA).

\section{Statistical Analyses}

The dependent variables of interest were the peak PFJFs and the peak PFJS (ie, the highest value throughout the duration of the squat). In addition, we tracked the vertical excursion of the center of mass of the pelvis using the motion capture system to assess potential differences in squat depth among the conditions. The pelvis center of mass was estimated based on the location of the markers placed on the anterior superior and posterior superior iliac spines. We identified the variables of interest for the final 3 trials for all conditions (baseline, FoAin, and FoAex). The first 2 trials were performed to allow participants to become acclimated to the new testing condition but were not analyzed. The 3-trial mean was calculated for each dependent variable, and a repeated-measures ANOVA was performed to analyze potential differences among the conditions. In the case of a significant omnibus test, Bonferroni post hoc tests were conducted. An alpha of .05 was used for all tests of statistical significance. We used SPSS software (version 24; IBM Corp, Armonk, NY) to perform our statistical analysis. Cohen's $d$ effect size statistic $(d)$ was calculated for each of the comparisons by dividing the mean difference by the average of the SDs for each condition. We evaluated the effect size statistics using the following criteria: trivial, 0 to 0.19 ; small, 0.20 to 0.49 ; medium, 0.50 to 0.79 ; and large, $\geq 0.80 .{ }^{35}$ The $90 \%$ confidence intervals $\left(\mathrm{CI}_{90 \%}\right)$ were also calculated for the differences in PFJFs and PFJS for each comparison.

As a follow-up analysis, we evaluated the consistency of the response to the verbal instructions by calculating the percent change for the FoAin and FoAex conditions in comparison with baseline for each participant. We also compared the change in PFJFs and PFJS for each participant with the minimal detectable change (MDC), which reflects the smallest difference that exceeds potential measurement error. (In this case, "error" likely reflects natural trial-to-trial variability.) To calculate the MDC, the intertrial consistency for the baseline trials was captured using Cronbach alpha coefficient $(\alpha){ }^{36}$ This coefficient was used to calculate the SEM (Equation 7), which was used to calculate the MDC for a 90\% confidence interval (MDC $90 \%$; Equation 8).

$$
\begin{gathered}
\mathrm{SEM}=\mathrm{SD} \times \sqrt{1-\alpha}, \\
\mathrm{MDC}_{90 \%}=1.645 \times \mathrm{SEM} \times \sqrt{2} .
\end{gathered}
$$

\section{Results}

The mean (SD) of age, mass, and height of the participants were $22.7(5.5)$ years, $64.3(8.5) \mathrm{kg}$, and $1.7(0.1) \mathrm{m}$, respectively. The median (range) time that participants reported engaging in weightbearing aerobic activity was 300 (120-600) minutes per week. All participants reported that running was their most common mode of physical activity. Each participant reported that they had experience performing the bodyweight squat exercise; however, only one participant reported that they had received formal training regarding their technique.

The ANOVA results indicated that there was not a significant difference in pelvis excursion among the testing conditions $(P=.60)$. As a result, any differences in knee mechanics were not due to a difference in squat depth. There was a difference among the conditions for the PFJFs $(P=.001)$ and the PFJS $(P<.001)($ Table 1$)$. The post hoc tests indicated that participants

\section{Table 1 Knee Mechanics for the Baseline Trials and Trials Performed Following Instruction Intended to Promote an FoAin or FoAex}

\begin{tabular}{lccc}
\hline & Baseline & FoAin & FoAex \\
\hline Patellofemoral mechanics & & & \\
PFJFs, N/kg & $35.4(5.9)$ & $31.3(5.0)^{*}$ & $32.3(5.4)^{*}$ \\
PFJS, mPa & $10.7(1.2)$ & $9.2(1.3)^{*}$ & \\
Model inputs & & $9.6(1.3)^{*}$ \\
Knee angles, deg & $99.5(7.3)$ & $93.3(9.0)$ & $96.0(10.8)$ \\
Knee moments, N.m/kg & $0.9(0.1)$ & $0.8(0.1)$ & $0.8(0.1)$ \\
\hline
\end{tabular}

Abbreviations: FoAin and FoAex, internal and external focus of attention, respectively; PFJFs, patellofemoral joint forces; PFJS, patellofemoral joint stress. Note: Data are presented as mean (SD).

*Significant difference versus baseline condition $(P<.05)$. 
demonstrated lower PFJFs for the FoAin $(P=.01 ; d=0.75$; $\mathrm{CI}_{90 \%},-6.08$ to -2.27$)$ and the FoAex $(P=.03 ; d=0.55$; $\mathrm{CI}_{90 \%},-4.92$ to -1.29$)$ conditions in comparison with baseline. They also demonstrated less PFJS for the FoAin $(P=.002$; $d=1.20 ; \mathrm{CI}_{90 \%},-2.06$ to -0.93$)$ and the FoAex $(P=.04 ; d=0.88$; $\mathrm{CI}_{90 \%},-1.66$ to -0.43 ) conditions (vs baseline). There was not a significant difference between the FoAin and FoAex conditions for the PFJFs $\left(P=.84 ; d=0.19 ; \mathrm{CI}_{90 \%},-2.76\right.$ to 0.63$)$ or the PFJS $\left(P=.41 ; d=0.31 ; \mathrm{CI}_{90 \%},-0.95\right.$ to 0.05$)$ variables. The peak kneeflexion angles and peak knee extension moments for each condition are also reported in Table 1.

For the FoAin condition, 5 participants (45.5\%) demonstrated a reduction in PFJFs that exceeded the MDC $(3.51 \mathrm{~N} / \mathrm{kg}$ ), and 7 participants $(63.6 \%)$ demonstrated a reduction in PFJS that exceeded the MDC $(1.02 \mathrm{mPa})$. For the FoAex condition, 5 participants $(45.5 \%)$ demonstrated a reduction in PFJFs that exceeded the MDC, and 8 participants $(72.7 \%)$ demonstrated a reduction in PFJS that exceeded the MDC. None of the participants demonstrated an increase in PFJFs or PFJS that exceeded the MDC for the FoAin or FoAex conditions (vs baseline). Individual participant responses to verbal instruction are presented in Table 2.

\section{Discussion}

The purpose of this study was to investigate how verbal instruction influences patellofemoral joint loading during bodyweight squatting and to compare the influence of instructions intended to promote an FoAin versus FoAex. Our results supported our primary hypothesis, as participants demonstrated a reduction in PFJFs and PFJS following verbal instruction, compared with baseline trials performed prior to instruction. This is encouraging, as it appears that relatively simple verbal instructions can influence squat mechanics in a manner that may reduce patellofemoral joint loading. However, we did not find that there was a difference in patellofemoral joint loading when participants received instructions intended to promote an FoAin versus FoAex, which did not

\section{Table 2 Individual Participant Responses Following Instruction Intended to Promote an FoAin or FoAex, Compared With Baseline Trials}

\begin{tabular}{|c|c|c|c|c|}
\hline \multirow[b]{2}{*}{ Participant } & \multicolumn{2}{|c|}{ FoAin vs baseline } & \multicolumn{2}{|c|}{ FoAex vs baseline } \\
\hline & PFJFs & $\overline{\text { PFJS }}$ & PFJFs & $\overline{\text { PFJS }}$ \\
\hline 1 & $-14.9^{\mathrm{a}}$ & $-14.6^{\mathrm{a}}$ & $-16.2^{\mathrm{a}}$ & $-16.1^{\mathrm{a}}$ \\
\hline 2 & $-26.8^{\mathrm{a}}$ & $-30.1^{\mathrm{a}}$ & -7.5 & $-10.1^{\mathrm{a}}$ \\
\hline 3 & -7.7 & $-13.9^{\mathrm{a}}$ & $-9.3^{\mathrm{a}}$ & $-9.1^{\mathrm{a}}$ \\
\hline 4 & 3.1 & 0.3 & 7.7 & 8.7 \\
\hline 5 & $-22.3^{\mathrm{a}}$ & $-24.2^{\mathrm{a}}$ & $-27.9^{\mathrm{a}}$ & $-29.6^{\mathrm{a}}$ \\
\hline 6 & -4.8 & -1.7 & 3.5 & 6.7 \\
\hline 7 & -6.5 & -7.1 & $-7.9^{\mathrm{a}}$ & $-10.2^{\mathrm{a}}$ \\
\hline 8 & $-11.2^{\mathrm{a}}$ & $-18.1^{\mathrm{a}}$ & $-16.2^{\mathrm{a}}$ & $-22.7^{\mathrm{a}}$ \\
\hline 9 & -10.3 & $-13.6^{\mathrm{a}}$ & -2.0 & -1.7 \\
\hline 10 & -4.6 & -7.0 & -7.4 & $-10.1^{\mathrm{a}}$ \\
\hline 11 & $-17.9^{\mathrm{a}}$ & $-21.2^{\mathrm{a}}$ & -7.7 & $-10.0^{\mathrm{a}}$ \\
\hline
\end{tabular}

Abbreviations: FoAin and FoAex, internal and external focus of attention, respectively; PFJFs, patellofemoral joint forces; PFJS, patellofemoral joint stress. Note: Percent change for FoAin and FoAex conditions versus baseline for each participant. Negative values indicate a decrease following instruction.

${ }^{a}$ Values indicate that the change exceeded the minimal detectable change. support our hypothesis. Our results appear to indicate the type of verbal instruction (ie, those intended to promote an FoAin vs FoAex) may not have a prominent influence on patellofemoral joint loading during the bodyweight squat exercise.

To our knowledge, this is the first study to analyze the influence of verbal instruction on patellofemoral joint loading during the bodyweight squat exercise. For both types of verbal instruction (FoAin and FoAex), participants demonstrated a medium or large reduction in PFJFs and PFJS compared with baseline (based on the effect size statistic). These results supplement earlier findings, which indicated that knee loading was reduced when anterior motion of the knees relative to the toes was restricted via guidewires ${ }^{16}$ or altered by manipulating foot position during squatting. ${ }^{37} \mathrm{~A}$ benefit of relying on simple verbal instruction is that this approach can be easily incorporated by exercise professionals who already typically utilize verbal cues to guide exercise technique. It appears that providing simple verbal instructions to limit anterior motion of the knees relative to the toes may reduce patellofemoral joint loading, which could potentially be useful in the management of patellofemoral pain.

Following instruction, almost half of the participants demonstrated a reduction in PFJFs and the majority of the participants demonstrated a reduction in PFJS, which exceeded potential measurement error (based on comparison of the changes relative to the MDC). In addition, none of the participants demonstrated an increase in PFJFs or PFJS that exceeded potential measurement error following instruction. As a result, we believe that exercise professionals can feel fairly comfortable providing instructions such as those described in this study, as our findings suggest that some/most individuals are likely to experience a reduction in patellofemoral loading with instruction and an increase in patellofemoral joint loading appears unlikely. We believe that analysis/ reporting of individual participant responses provides valuable insight, as it indicates the proportion of individuals that can be expected to demonstrate a positive mechanical response to intervention (in this case, a reduction in patellofemoral joint loading with verbal instruction). ${ }^{38}$ However, it is important to note that our analysis does not indicate the proportion of individuals that demonstrated a "clinically meaningful" change in patellofemoral loading, as our analysis only tested uninjured participants during a single session.

We anticipated that participants would demonstrate a more prominent reduction in patellofemoral joint loading when they were given instructions intended to promote an FoAex, in comparison with trials performed following instructions intended to promote an FoAin. However, our findings did not support this hypothesis as there was no difference in PFJFs or PFJS between the FoAin and FoAex conditions. Based on our findings, it appears that both types of verbal instruction reduced patellofemoral joint loading to a similar extent. However, it is important to note that our analysis only indicates that there was not a marked difference in performance between the FoAin and FoAex conditions, as this was not a longitudinal training study where retention could be evaluated. Future studies should be conducted to determine how different types of exercise instruction influence retention within and between sessions. ${ }^{17}$ In addition, our analysis only focused on one aspect of performance (ie, patellofemoral joint loading). It is possible that there were differences among the conditions that were not investigated as part of this study (eg, changes in other mechanical variables, differences in muscle activity).

Although we believe that our findings make a valuable contribution to the literature, the limitations of this study need 
to be considered. First, our study only included participants who were currently free from injury. As a result, we are unable to ascertain how our findings generalize to individuals with patellofemoral pain. Considering this limitation, our results are potentially more relevant to patellofemoral pain prevention (either the initial occurrence or the recurrence). In addition, we only analyzed how verbal instruction influenced patellofemoral joint loading. Exercise professionals must take into consideration how altering squat technique may influence the entire kinetic chain. ${ }^{39,40}$ Also, we did not standardize squat rate during testing (eg, via metronome). We did consider incorporating this into our testing protocol; however, we were concerned that it may limit a participant's ability to attend to exercise instruction, which we believed was a more critical aspect of this study. Our study is also limited by the fact that ground reaction forces were only recorded for the dominant limb. This requires us to assume that participants maintained similar weight-bearing symmetry across testing conditions. However, we believe that this is a reasonable assumption because the instructions were not specific to the limb that was evaluated (ie, participants were given general instructions to limit anterior knee motion, which pertained to both limbs). In addition, it is also important to acknowledge the relatively small sample size included in this study. Although we conducted an a priori sample size estimate, this was related to the omnibus test (repeated-measures ANOVA). As a result, our study may not have been adequately powered to identify more subtle differences between the FoAin and FoAex conditions. However, it is important to note that the magnitude of the differences in PFJFs and PFJS between the FoAin and FoAex conditions was considered trivial and small based on the effect sizes, and the CIs for these differences included zero (indicative of nonsignificant differences). Finally, we recommend that future studies quantify trunk position, as this may influence knee/patellofemoral loading. ${ }^{41,42}$

In addition to the limitations associated with the study, it is also important to highlight the limitations associated with our patellofemoral joint model. First, it should be noted that the model used in this study only incorporates sagittal plane mechanics. Although the bodyweight squat exercise is comprised predominantly of movement in the sagittal plane, frontal and transverse plane mechanics may also influence patellofemoral joint loading. Also, our model relies on net joint moments calculated via inverse dynamics, which does not account for muscle cocontraction. The model used in this study was consistent across all testing conditions. However, it is possible that the inability to account for cocontraction may not have had a consistent influence among the conditions, as it appears that attentional focus may influence muscle cocontraction. ${ }^{43}$ Finally, subtle changes to the model, such as altering the equation used to estimate patellofemoral contact area, could have a prominent influence on the results of our analysis. ${ }^{44}$ This is a challenge associated with estimating patellofemoral joint loading in vivo; however, we certainly believe that attempts to estimate tissue-specific loading via modeling provide valuable insight. Regardless, care must be taken when comparing the results of this study with other analyses that relied on slightly different methodological approaches.

\section{Conclusion}

Young, active females demonstrated a reduction in patellofemoral joint loading during squatting when they were given verbal instruction intended to limit anterior knee motion relative to the toes, compared with trials performed prior to instruction. However, there was not a prominent difference in patellofemoral joint loading when they received verbal instructions intended to promote an FoAin versus FoAex. It appears that simple verbal instructions regarding squat technique are sufficient to reduce patellofemoral joint loading. As a result, it is possible that instructions of this nature may be beneficial when prescribing the squat exercise for treatment or prevention of patellofemoral pain.

\section{Acknowledgments}

This project was not supported by grant funding. Each of the authors listed has met the criteria for authorship. The authors do not have any conflicts of interest to report.

\section{References}

1. Boling M, Padua D, Marshall S, Guskiewicz K, Pyne S, Beutler A. Gender differences in the incidence and prevalence of patellofemoral pain syndrome. Scand J Med Sci Sports. 2010;20(5):725-730. PubMed ID: 19765240 doi:10.1111/j.1600-0838.2009.00996.x

2. Taunton JE, Ryan MB, Clement DB, McKenzie DC, Lloyd-Smith DR, Zumbo BD. A retrospective case-control analysis of 2002 running injuries. Br J Sports Med. 2002;36(2):95-101. PubMed ID: 11916889 doi:10.1136/bjsm.36.2.95

3. Rathleff MS, Rathleff CR, Olesen JL, Rasmussen S, Roos EM. Is knee pain during adolescence a self-limiting condition? Prognosis of patellofemoral pain and other types of knee pain. Am J Sports Med. 2016;44(5):1165-1171. PubMed ID: 26792702 doi:10.1177/ 0363546515622456

4. Conchie H, Clark D, Metcalfe A, Eldridge J, Whitehouse M. Adolescent knee pain and patellar dislocations are associated with patellofemoral osteoarthritis in adulthood: a case control study. Knee. 2016;23(4):708-711. PubMed ID: 27180253 doi:10.1016/j.knee. 2016.04.009

5. Utting MR, Davies G, Newman JH. Is anterior knee pain a predisposing factor to patellofemoral osteoarthritis? Knee. 2005;12(5): 362-365. PubMed ID: 16146626 doi:10.1016/j.knee.2004.12.006

6. Brechter J, Powers CM. Patellofemoral stress during walking in persons with and without patellofemoral pain. Med Sci Sports Exerc. 2002;34(10):1582-1593. doi:10.1097/00005768-200210000-00009

7. Farrokhi S, Keyak JH, Powers CM. Individuals with patellofemoral pain exhibit greater patellofemoral joint stress: a finite element analysis study. Osteoarthritis Cartilage. 2011;19(3):287-294. PubMed ID: 21172445 doi:10.1016/j.joca.2010.12.001

8. Peng HT, Chen WC, Kernozek TW, Kim K, Song CY. Influences of patellofemoral pain and fatigue in female dancers during ballet jump-landing. Int J Sports Med. 2015;36(9):747-753. PubMed ID: 25806586 doi:10.1055/s-0035-1547220

9. Thomeer LT, Sheehan FT, Jackson JN. Normalized patellofemoral joint reaction force is greater in individuals with patellofemoral pain. J Biomech. 2017;60:238-242. PubMed ID: 28755815 doi:10.1016/j. jbiomech.2017.06.024

10. Kooiker L, Van De Port IG, Weir A, Moen MH. Effects of physical therapist-guided quadriceps-strengthening exercises for the treatment of patellofemoral pain syndrome: a systematic review. J Orthop Sports Phys Ther. 2014;44(6):391-B1. PubMed ID: 24766358 doi:10. 2519/jospt.2014.4127

11. Bolgla L, Malone T. Exercise prescription and patellofemoral pain: evidence for rehabilitation. $J$ Sport Rehabil. 2005;14:72-88. doi:10. 1123/jsr.14.1.72

12. Wallace DA, Salem GJ, Salinas R, Powers CM. Patellofemoral joint kinetics while squatting with and without an external load. J Orthop 
Sports Phys Ther. 2002;32(4):141-148. PubMed ID: 11949662 doi:10.2519/jospt.2002.32.4.141

13. Chandler J, McMillan J, Kibler WB, Richards D. Safety of the squat exercise. ACSM Curr Comment. 2002.

14. Sands WA, Wurth JJ, Hewit JK. Basics of Strength and Conditioning Manual. Colorado Springs, CO: National Strength and Conditioning Association; 2012.

15. Schoenfeld BJ. Squatting kinematics and kinetics and their application to exercise performance. J Strength Cond Res. 2010;24(12):34973506. PubMed ID: 20182386 doi:10.1519/JSC.0b013e3181bac2d7

16. Kernozek TW, Gheidi N, Zellmer M, Hove J, Heinert BL, Torry MR. Effects of anterior knee displacement during squatting on patellofemoral joint stress. J Sport Rehabil. 2018;27(3):237-243. doi:10.1123/ jsr.2016-0197

17. Hunt C, Paez A, Folmar E. The impact of attentional focus on the treatment of musculoskeletal and movement disorders. Int J Sports Phys Ther. 2017;12(6):901-907. PubMed ID: 29158952 doi:10. 26603/ijspt20170901

18. Wulf G, Shea C, Lewthwaite R. Motor skill learning and performance: a review of influential factors. Med Educ. 2010;44(1):75-84. PubMed ID: 20078758 doi:10.1111/j.1365-2923.2009.03421.x

19. Durham K, Van Vliet PM, Badger F, Sackley C. Use of information feedback and attentional focus of feedback in treating the person with a hemiplegic arm. Physiother Res Int. 2009;14(2):77-90. PubMed ID: 19107706 doi:10.1002/pri.431

20. Johnson L, Burridge JH, Demain SH. Internal and external focus of attention during gait re-education: an observational study of physical therapist practice in stroke rehabilitation. Phys Ther. 2013;93(7): 957-966. PubMed ID: 23559523 doi:10.2522/ptj.20120300

21. Abdollahipour R, Wulf G, Psotta R, Palomo Nieto M. Performance of gymnastics skill benefits from an external focus of attention. J Sports Sci. 2015;33(17):1807-1813. PubMed ID: 25774536 doi:10.1080/ 02640414.2015.1012102

22. Halperin I, Williams KJ, Martin DT, Chapman DW. The effects of attentional focusing instructions on force production during the isometric midthigh pull. J Strength Cond Res. 2016;30(4):919-923. PubMed ID: 27003451 doi:10.1519/JSC.0000000000001194

23. Porter JM, Nolan RP, Ostrowski EJ, Wulf G. Directing attention externally enhances agility performance: a qualitative and quantitative analysis of the efficacy of using verbal instructions to focus attention. Front Psychol. 2010;1:216. PubMed ID: 21833271 doi:10. 3389/fpsyg.2010.00216

24. Schutts KS, Wu WFW, Vidal AD, Hiegel J, Becker J. Does focus of attention improve snatch lift kinematics? J Strength Cond Res. 2017;31(10):2758-2764. PubMed ID: 28030531 doi:10.1519/JSC. 0000000000001753

25. Wulf G, Dufek JS, Lozano L, Pettigrew C. Increased jump height and reduced EMG activity with an external focus. Hum Mov Sci. 2010; 29(3):440-448. PubMed ID: 20409600 doi:10.1016/j.humov.2009. 11.008

26. Cohen J. Statistical Power Analysis for the Behavioral Sciences (2nd ed.). Hillsdale, NJ: L. Erlbaum Associates; 1988.

27. Faul F, Erdfelder E, Lang AG, Buchner A. G*Power 3: a flexible statistical power analysis program for the social, behavioral, and biomedical sciences. Behav Res Methods. 2007;39(2):175-191. PubMed ID: 17695343 doi:10.3758/BF03193146

28. Benjaminse A, Gokeler A, Dowling AV, et al. Optimization of the anterior cruciate ligament injury prevention paradigm: novel feedback techniques to enhance motor learning and reduce injury risk. J Orthop Sports Phys Ther. 2015;45(3):170-182. PubMed ID: 25627151 doi:10.2519/jospt.2015.4986
29. Bell AL, Pedersen DR, Brand RA. Prediction of hip joint center location from external landmarks. Hum Mov Sci. 1989;8:3-16. doi:10.1016/0167-9457(89)90020-1

30. Bell AL, Pedersen DR, Brand RA. A comparison of the accuracy of several hip center location prediction methods. J Biomech. 1990;23(6): 617-621. PubMed ID: 2341423 doi:10.1016/0021-9290(90)90054-7

31. Grood ES, Suntay WJ. A joint coordinate system for the clinical description of three-dimensional motions: application to the knee. $J$ Biomech Eng. 1983;105(2):136-144. PubMed ID: 6865355 doi:10. 1115/1.3138397

32. Dempster WT. Space requirements of the seated operator: geometrical, kinematic, and mechanical aspects of the body with special reference to the limbs. Technical Report WADC-TR-55-159. 1955;55-159.

33. van Eijden TM, Weijs WA, Kouwenhoven E, Verburg J. Forces acting on the patella during maximal voluntary contraction of the quadriceps femoris muscle at different knee flexion/extension angles. Acta Anat. 1987;129(4):310-314. PubMed ID: 3630619 doi:10.1159/ 000146421

34. Powers CM, Lilley JC, Lee TQ. The effects of axial and multiplane loading of the extensor mechanism on the patellofemoral joint. Clin Biomech. 1998;13(8):616-624. doi:10.1016/S0268-0033(98) 00013-8

35. Cohen J. A power primer. Psychol Bull. 1992;112(1):155-159. PubMed ID: 19565683 doi:10.1037/0033-2909.112.1.155

36. Varghese R, Hui-Chan CWY, Wang E, Bhatt T. Internal consistency and test-retest reliability of an instrumented functional reaching task using wireless electromyographic sensors. J Electromyogr Kinesiol. 2014;24:593-600. PubMed ID: 25026882 doi:10.1016/j.jelekin. 2014.05.011

37. Escamilla RF, Zheng N, Macleod TD, et al. Patellofemoral joint force and stress during the wall squat and one-leg squat. Med Sci Sports Exerc. 2009;41(4):879-888. PubMed ID: 19276845 doi:10.1249/ MSS.0b013e31818e7ead

38. Barrios J, Willson J. Minimum detectable change in medial tibiofemoral contact force parameters: derivation and application to a loadaltering intervention. J Appl Biomech. 2017;33(2):171-175. PubMed ID: 27834573 doi:10.1123/jab.2016-0163

39. Fry AC, Smith JC, Schilling BK. Effect of knee position on hip and knee torques during the barbell squat. J Strength Cond Res. 2003; 17(4):629-633. PubMed ID: 14636100

40. Lorenzetti S, Gulay T, Stoop M, et al. Comparison of the angles and corresponding moments in the knee and hip during restricted and unrestricted squats. J Strength Cond Res. 2012;26(10):2829-2836. PubMed ID: 22801421 doi:10.1519/JSC.0b013e318267918b

41. Farrokhi S, Pollard CD, Souza RB, Chen Y, Reischl S, Powers CM. Trunk position influences the kinematics, kinetics, and muscle activity of the lead lower extremity during the forward lunge exercise. J Orthop Sports Phys Ther. 2008;38(7):403-409. PubMed ID: 18591759 doi:10.2519/jospt.2008.2634

42. Teng H, Powers CM. Sagittal plane trunk posture influences patellofemoral joint stress during running. J Orthop Sports Phys Ther. 2014;44(10):785-792. PubMed ID: 25155651 doi:10.2519/jospt. 2014.5249

43. Lohse KR, Sherwood DE. Thinking about muscles: the neuromuscular effects of attentional focus on accuracy and fatigue. Acta Psychol. 2012;140(3):236-245. doi:10.1016/j.actpsy.2012.05.009

44. Nunes GS, Silva RS, dos Santoa AF, Fernandes RAS, Serrao FV, de Noronha M. Methods to assess patellofemoral joint stress: a systematic review. Gait Posture. 2018;61:188-196. PubMed ID: 29353744 doi:10.1016/j.gaitpost.2017.12.018 